\title{
Effects of Beam-beam Interaction on Spin Motion
}

\author{
A. Luccio, M. Syphers
}

November 25, 1997

To begin to study the effects of the beam-beam interaction on polarization in RHIC, the SPINK[1] code has been updated to allow for head-on interactions at specified collision points. The interaction is calculated for an individual particle of arbitrary betatron amplitude passing through an on-coming round Gaussian beam of specified emittance.

Consider a single proton passing through a round on-coming proton bunch which has rms transverse size $\sigma$, and longitudinal proton density $\lambda(s)$ (particles per meter). The force experienced by the single test particle within the distribution is purely radial due to the symmetry of the problem, and is given by

$$
\begin{aligned}
F_{r}(r, s) & =\frac{\lambda(s) e^{2}}{2 \pi \epsilon_{0}}\left(1+\frac{v^{2}}{c^{2}}\right) \frac{1-e^{-r^{2} / 2 \sigma^{2}}}{r} \\
& \approx \frac{\lambda(s) e^{2}}{2 \pi \epsilon_{0} \sigma^{2}} r
\end{aligned}
$$

where $r$ is the transverse radial distance from the center of the bunch, and $e$ is the proton charge. The last expression is for $r<\sigma$. The beam-beam interaction, for most of the protons in the center of the bunch, has a defocusing effect. For high energy collisions, $v \approx c$ and so the quantity in parentheses is approximately equal to 2 . In fact, if the space charge force due to the the surrounding protons of the test particle's own equally populated bunch is included, the factor is exactly 2 , and so we will use this value from now on.

The horizontal $(x)$ and vertical $(y)$ components of the force may thus be written as

$$
\begin{aligned}
& F_{x}=\frac{\lambda(s) e^{2}}{2 \pi \epsilon_{0} \sigma^{2}} x \mathcal{F}(r), \\
& F_{y}=\frac{\lambda(s) e^{2}}{2 \pi \epsilon_{0} \sigma^{2}} y \mathcal{F}(r),
\end{aligned}
$$

where

$$
\mathcal{F}(r) \equiv \frac{1-e^{-r^{2} / 2 \sigma^{2}}}{\left(\frac{r^{2}}{2 \sigma^{2}}\right)}, \quad r^{2} \equiv x^{2}+y^{2}
$$




\section{Effects on Particle Trajectory}

We apply a "thin lens" approach to describing the interaction of the test particle with the on-coming bunch. The transverse displacement of the particle is not altered, while the slope of its trajectory changes according to

$$
\begin{aligned}
\Delta x^{\prime} & =\frac{\int F_{x} d t}{p}=\frac{\int F_{x} d z / v}{p} \\
& =\frac{e^{2}}{2 \pi \epsilon_{0} \gamma m c^{2}} \frac{x}{\sigma^{2}} \mathcal{F}(r) \int \lambda(z) d z \\
& =\frac{e^{2}}{4 \pi \epsilon_{0} m c^{2}} \frac{n x}{\gamma \sigma^{2}} \mathcal{F}(r)
\end{aligned}
$$

where we assume $v \approx c$, and we have used the fact that $\int \lambda(z) d z=n / 2, n$ being the total number of particles in the on-coming bunch. The factor of one-half comes from the fact that the test particle and the bunch are moving toward each other.

Written in terms of the $95 \%$ normalized emittance of the on-coming bunch, the test particle's horizontal and vertical slopes are changed by amounts

$$
\begin{aligned}
\Delta x^{\prime} & =\frac{6 \pi r_{0} n}{\beta^{*} \epsilon_{N}} x \mathcal{F}(r), \\
\Delta y^{\prime} & =\frac{6 \pi r_{0} n}{\beta^{*} \epsilon_{N}} y \mathcal{F}(r),
\end{aligned}
$$

with $r_{0}$ being the classical radius of the proton, $r_{0}=1.53 \times 10^{-18} \mathrm{~m}$, and $\beta^{*}$ is the amplitude function at the interaction point.

Also in this approximation, we are assuming that the bunch length is much smaller than $\beta^{*}$ which permits us to neglect hour-glass effects. This is in accord with our "thin lens" treatment, for if we look at particles with small betatron amplitudes (for which $\mathcal{F}$ is near unity) then the effective focal length of the "lens"

$$
f^{*} \equiv \frac{\beta^{*} \epsilon_{n}}{6 \pi r_{0} n}
$$

is on the order of $100 \mathrm{~m}$ for standard RHIC polarized beam parameters $\left(\beta^{*}=1 \mathrm{~m}\right.$, $\left.\epsilon_{N}=20 \pi \mathrm{mm}-\mathrm{mr}, n=2 \times 10^{11}\right)$. The expected $\mathrm{rms}$ bunch length for proton beams in RHIC is $60 \mathrm{~cm}$ or less.

The equivalent focal length, $f^{*}$, leads to the familiar beam-beam tune shift for small amplitude oscillations

$$
\xi \equiv \frac{1}{4 \pi} \frac{\beta^{*}}{f^{*}}=\frac{3 r_{0} n}{2 \epsilon_{N}} .
$$

The beam-beam tune shift for the beam parameters used above is $\xi=0.007$ per crossing, or 0.014 for two IRs. 


\section{Effects on Particle Spin}

We start with the results of Luccio[2]. If the equation of motion of the spin vector is written as $d \vec{S} / d t=-\vec{\Omega} \times \vec{S}$, then the transformation of the spin vector through arbitrary (but constant) electromagnetic fields over a small time interval $\Delta t$ can be given by a rotation matrix $R$

$$
\begin{aligned}
\vec{S} & =R \vec{S}_{0}, \\
R & =I \cos \phi+W\left(\frac{1-\cos \phi}{\omega^{2}}\right)+A\left(\frac{\sin \phi}{\omega}\right)
\end{aligned}
$$

where

$$
W=\left(\begin{array}{ccc}
\Omega_{x}^{2} & \Omega_{x} \Omega_{y} & \Omega_{x} \Omega_{z} \\
\Omega_{x} \Omega_{y} & \Omega_{y}^{2} & \Omega_{y} \Omega_{z} \\
\Omega_{x} \Omega_{z} & \Omega_{y} \Omega_{z} & \Omega_{z}^{2}
\end{array}\right), \quad A=\left(\begin{array}{ccc}
0 & \Omega_{z} & -\Omega_{y} \\
-\Omega_{z} & 0 & \Omega_{x} \\
\Omega_{y} & -\Omega_{x} & 0
\end{array}\right),
$$

$\omega^{2} \equiv|\Omega|^{2}$, and $\phi \equiv \omega \Delta t$. For our case, $\Omega$ is given by the BMT equation[3], from which

$$
\vec{\Omega}=\frac{e}{m \gamma}\left\{G \gamma \vec{B}-G(\gamma-1) \frac{(\vec{v} \cdot \vec{B}) \vec{v}}{v^{2}}+\gamma\left[G-\frac{1}{\gamma^{2}-1}\right] \frac{\vec{E} \times \vec{v}}{c^{2}}\right\} .
$$

For the beam-beam interaction at high energies, $\phi$ is thus

$$
\begin{aligned}
\phi=\int \omega d t & =\int \frac{e}{m \gamma} G \gamma \frac{F_{r}(r, s)}{e v} d s \\
& =\frac{G r_{0} n}{\sigma^{2}} r \mathcal{F}(r) \\
& =\frac{6 \pi G \gamma r_{0} n}{\epsilon_{N} \beta^{*}} r \mathcal{F}(r) .
\end{aligned}
$$

Additionally, $\Omega_{x} / \Omega=y / r, \Omega_{y} / \Omega=-x / r$, and $\Omega_{z} / \Omega=0$ since the force is radial. Hence, the spin rotation matrix for the beam-beam interaction is

$$
R=I \cos \phi+\frac{1}{r^{2}}\left(\begin{array}{ccc}
y^{2} & -x y & 0 \\
-x y & x^{2} & 0 \\
0 & 0 & 0
\end{array}\right)(1-\cos \phi)+\frac{1}{r}\left(\begin{array}{ccc}
0 & 0 & x \\
0 & 0 & y \\
-x & -y & 0
\end{array}\right) \sin \phi .
$$

We note that $R \rightarrow I$ as $r \rightarrow 0$, and that $\operatorname{det} R=1$.

\section{Implementation and Tracking Results}

The effect of the beam-beam interaction on the particle spin is expected to be small. To see this, consider a particle with only vertical displacement as it passes through the 
on-coming bunch. If the particle's displacement is equal to the beam's rms amplitude, then the spin will precess by an amount

$$
\Delta \phi \approx G \gamma \Delta y^{\prime} \approx \frac{G r_{0} n}{\sigma^{*}}=G r_{0} n \sqrt{\frac{6 \pi \gamma}{\beta^{*} \epsilon_{N}}}
$$

which, for $2 \times 10^{11}$ particles per bunch, $20 \pi \mathrm{mm}$ mrad emittance, and $\beta^{*}=1 \mathrm{~m}$, corresponds to $\Delta \phi=5 \mathrm{mrad}$. This precession will contribute to the intrinsic depolarizing resonance strength at storage. Since the resonance strength is given by

$$
\epsilon_{k}=\frac{1+G \gamma}{2 \pi} \oint \sqrt{\frac{\beta(s) \epsilon_{N}}{6 \pi \gamma}} \frac{\partial B_{x}(s) / \partial y}{B \rho} e^{i k \theta(s)} d s,
$$

then to check the magnitude of the effect we compare $\sqrt{\beta^{*}} / f^{*}$ with the value $\sqrt{\beta_{\max }} / F$ for a standard RHIC arc quadrupole. We find that the contribution to the intrinsic resonance strength of the beam-beam interaction is roughly $14 \%$ of the contribution from a single RHIC arc quad for the parameters above.

From Eqs. 9, 10, 19, and 20, the orbit effects of the beam-beam interaction are governed by the bunch intensity, the beam emittance, and $\beta^{*}$, while the spin effects depend upon these parameters plus the beam energy. In the studies described below, we have assumed large beam-beam forces corresponding to on-coming bunches with emittances of $10 \pi \mathrm{mm}-\mathrm{mrad}$, intensity $2 \times 10^{11}$ protons per bunch, and $\beta^{*}$ of 1 meter. These parameters correspond to a beam-beam tune shift parameter of magnitude $\xi=0.015$ for one interaction point.

With beam-beam introduced into SPINK, the first test of the code was to see if the particle tracking showed the correct particle tune shift. A particle with near-zero betatron amplitude should see its tune shifted from the nominal accelerator tune by an amount $-\xi$, where a proton-proton collider generates a negative tune shift. A particle with a larger betatron amplitude, $a$, should see its tune shifted by an amount

$$
\Delta \nu(u)=-\frac{\xi}{(u / 2)^{2}}\left[1-e^{-(u / 2)^{2}} I_{0}\left[(u / 2)^{2}\right]\right]
$$

where $u \equiv a / \sigma$. Fig. 1 shows the above equation and tracking results for the beam parameters given above. The tracking agrees very well with the prediction.

Several other studies were performed using the new version of the code. In particular, particles were tracked for various constant energies in RHIC, and other simulations were performed in which particles were accelerated and then stored at a flat-top energy of $250 \mathrm{GeV}$. Discussions and results of these two cases are presented below.

\subsection{Spin Tracking in Storage Mode}

The SPINK code was used to track particles at constant energy and observe the evolution of the average and spread of the spin distribution. For this study, ten particles 


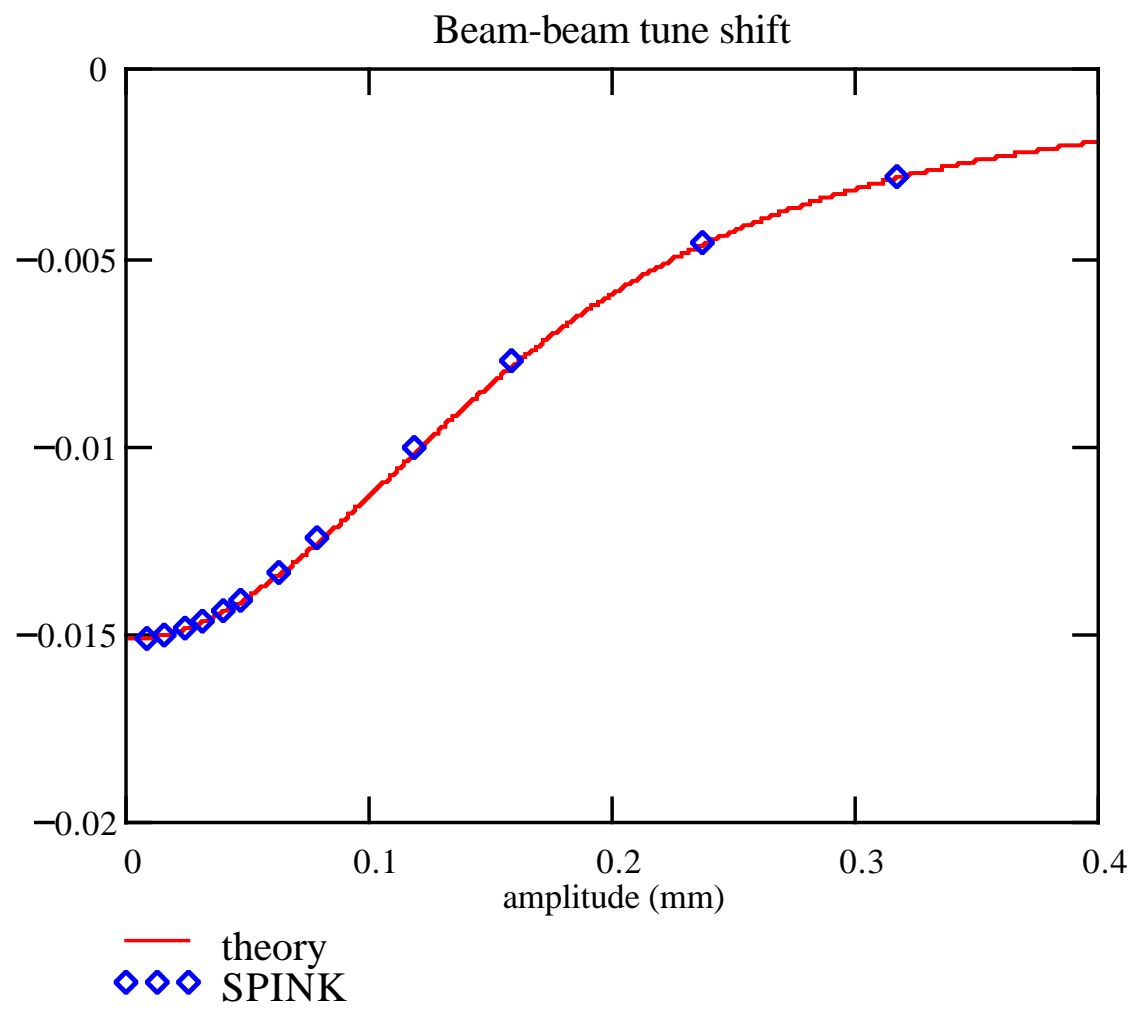

Figure 1: Beam-beam tune shift from theory (solid line) and from SPINK tracking results for one interaction point. The beam emittance is $10 \pi \mathrm{mm}-\mathrm{mr}$ and the bunch intensity is $2 \times 10^{11}$ particles per bunch. The rms beam size at the interaction point is $0.08 \mathrm{~mm}$. 


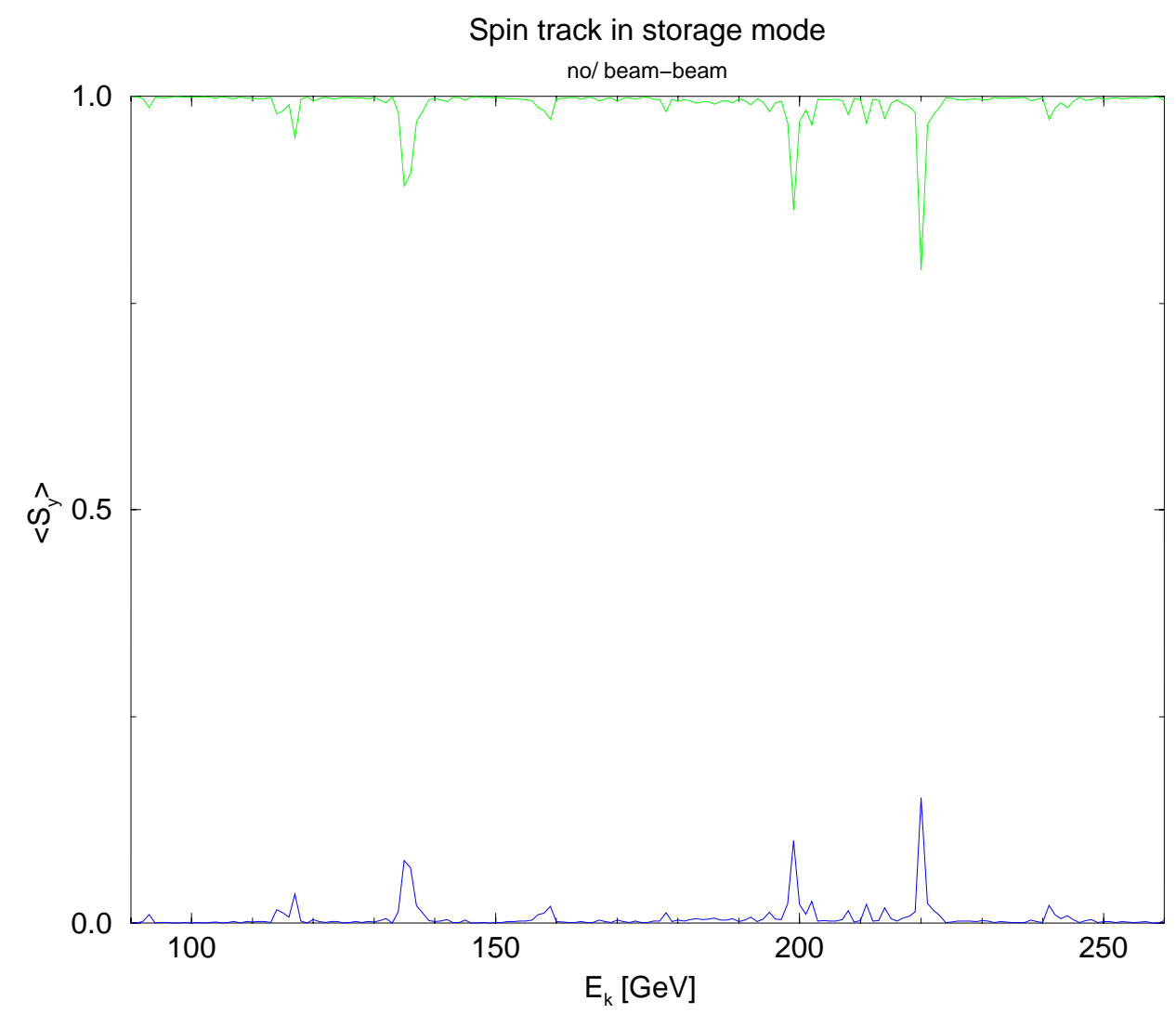

Figure 2: Final polarization achieved after tracking 10,000 turns at the given energy. The beam-beam interaction was turned off.

of various betatron amplitudes were tracked for 10,000 turns with the beam-beam interaction turned off and then re-tracked with beam-beam turned on assuming $2 \times 10^{11}$ particles in the on-coming bunch. The distribution was tracked at kinetic energies from $90 \mathrm{GeV}$ to $250 \mathrm{GeV}$ in $1 \mathrm{GeV}$ steps for both situations. The Siberian Snakes in RHIC were on during these simulations. The final results for the vertical spin component were averaged with weights reflecting the amplitude of each particle in the beam. The initial spin direction was assumed vertical, and the beam shape Gaussian corresponding to a normalized emittance of $10 \pi \mathrm{mm}$-mrad. Only one collision region was assumed. The bare tunes (i.e. with no beam-beam interaction) of the RHIC lattice was $\nu_{x}=28.19, \nu_{y}=29.18$.

The results of this study are shown in Figs. 2 and 3. The upper curve in both figures gives the polarization (vertical spin component), first averaged over the turns and then averaged with weights over the 10 particles; the lower curve gives the rms value of the weighted distribution, proportional (for small values) to the spin cone 


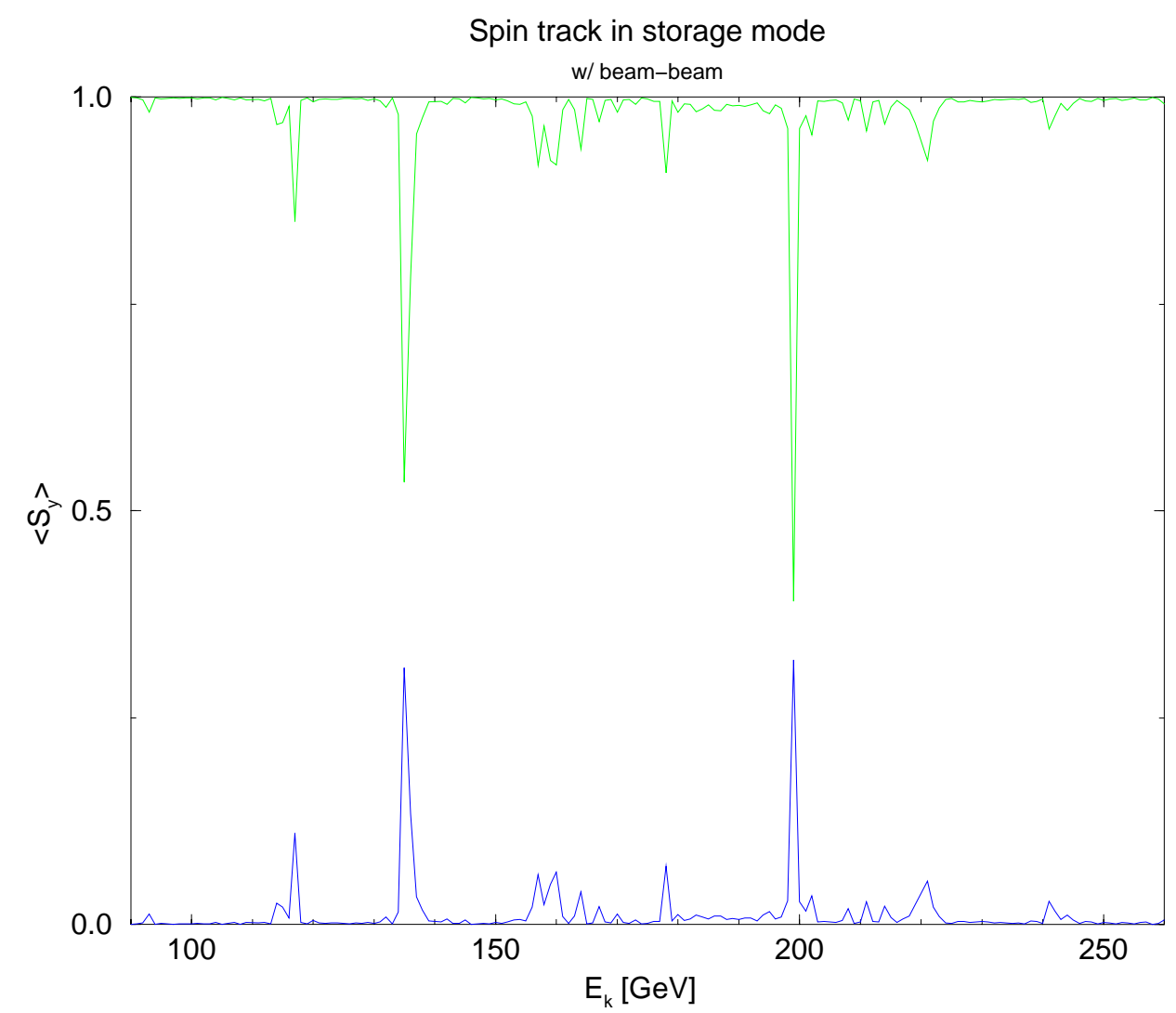

Figure 3: Same as previous figure, but with beam-beam interaction enabled. 
opening angle. No significant differences in the results were found when tracking for many more turns.

Fig. 2 shows some loss of polarization at selected energies, notably at kinetic energies of about 135, 200, and $220 \mathrm{GeV}$, where strong spin resonances are present. Fig. 3 shows a more pronounced loss of polarization at some locations, the largest at $\sim 200 \mathrm{GeV}$. It should be pointed out that tracking with acceleration through the same range of energy showed that with Snakes on full polarization is recovered after each resonance. This will be described later.

\subsection{Spin Tracking with Acceleration and Storage}

In the study described above, all particles were started with their spins aligned vertically. In RHIC, and in the presence of the beam-beam interaction, this may not be the proper closed spin direction. To better prepare the initial particle spin distribution for tracking at storage energy, spin tracking was started below $200 \mathrm{GeV}$ and particles were tracked to $250 \mathrm{GeV}$, thus allowing an equilibrium situation to be attained prior to long-term tracking at flat-top. The particle distribution was tracked from $\sim 157 \mathrm{GeV}(G \gamma=300)$ to $250 \mathrm{GeV}(G \gamma=479.4878)$ with no beam-beam interaction, and then stored at $250 \mathrm{GeV}$ with beam-beam on. The acceleration stage from $G \gamma=$ 300 to 470 took approximately 350,000 turns at an acceleration rate roughly 10 times faster than will be found in RHIC. Again, the purpose, however, was to prepare a spin distribution, not to look at acceleration effects in RHIC. This step was followed by a 45,000-turn segment during which the synchronous phase was shifted to $180^{\circ}$ by the time the beam energy reached $250 \mathrm{GeV}$. Also, during this time the beam-beam interaction was adiabatically turned on. After these steps, the particles were tracked for approximatly 15,000 turns. Fig. 4 shows the evolution of the vertical spin component for 12 particles with initial vertical displacements from the equilibrium orbit (at the interaction point) ranging from 0.01 to $0.40 \mathrm{~mm}$.

Fig. 5 shows the distribution of the vertical tune shift among the particles, and the statistical weights used to average, at $250 \mathrm{GeV}$. Table 1 shows the data used for Fig. 5 plus the polarization and its rms spread averaged for each particle at $250 \mathrm{GeV}$ over the 15,000 turns of storage. The table gives also the weighted average tune shift, average polarization and rms polarization for the distribution. Fig. 6 gives the average vertical spin component versus turn number for storage at $250 \mathrm{GeV}$ in the presence of the beam-beam interaction.

\subsection{Effects of Machine Detuning during Storage.}

Depolarizing resonances are enhanced when the fractional part of the vertical betatron tune approaches a ratio of two small integers. In RHIC, the fractions $1 / 6$ and $5 / 6$ are particularly dangerous. SPINK allows one to change the bare tune of the machine by a small amount (without running the optics, say Mad, over and over again) by 

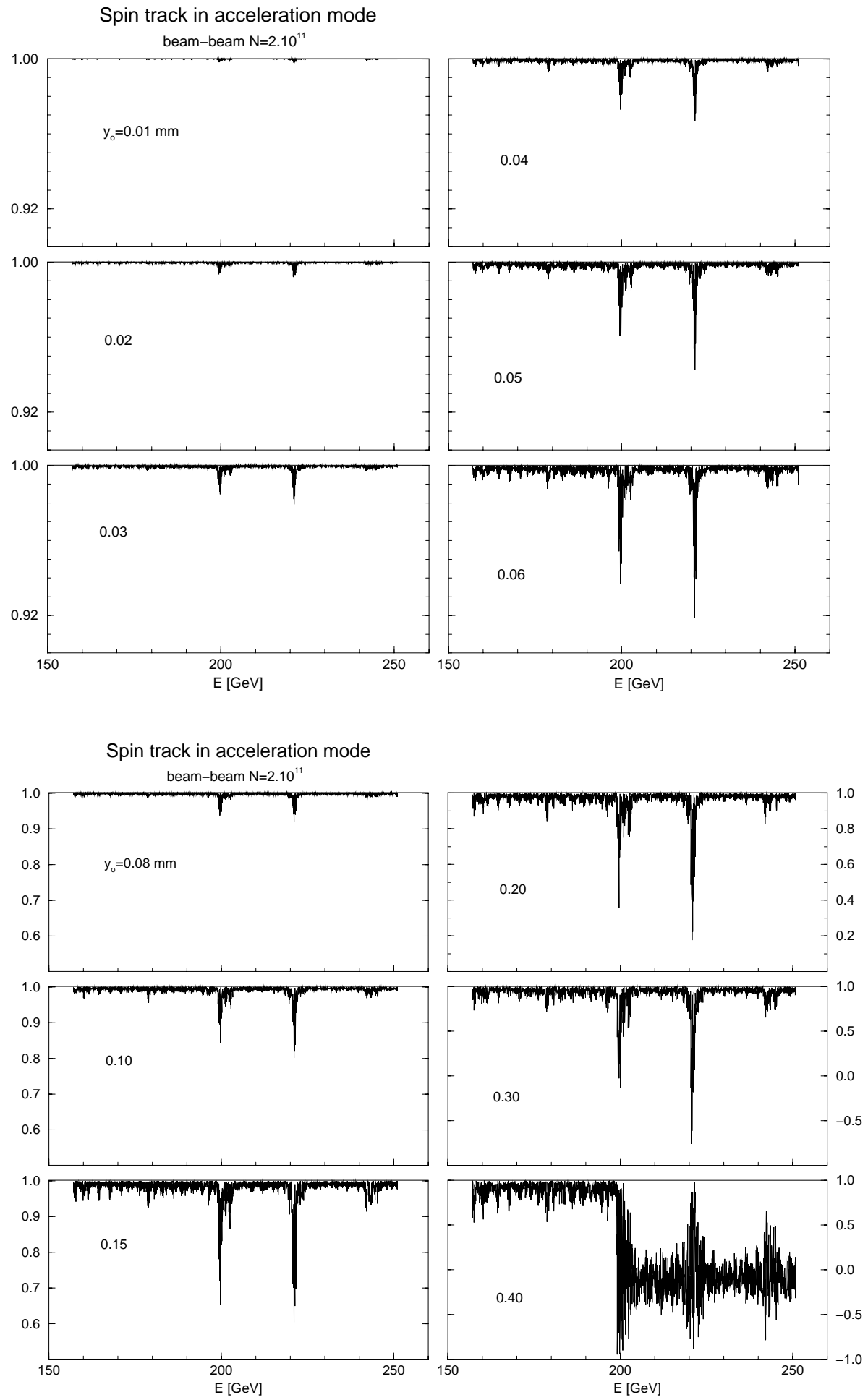

Figure 4: Tracking results with beam-beam interaction after acceleration to $250 \mathrm{GeV}$. Initial particle displacements at the interaction point range from $0.01 \mathrm{~mm}$ to $0.40 \mathrm{~mm}$. 


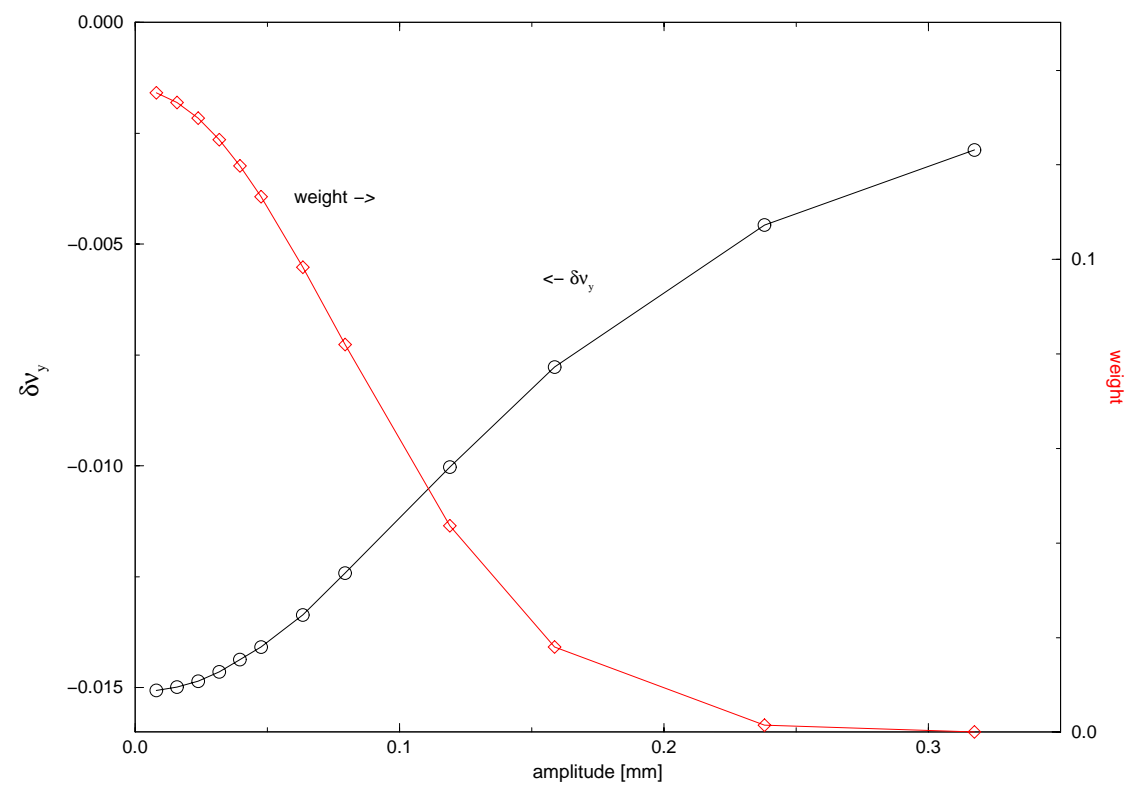

Figure 5: Tune shift analysis of tracking results, and the particle weights (assuming a Gaussian distribution) used for calculating moments.

\begin{tabular}{|c|c|c|c|c|}
\hline $\begin{array}{c}a_{0}, \mathrm{~mm} \\
(\text { at } 157 \mathrm{GeV})\end{array}$ & $\delta \nu_{y}$ & $\left\langle S_{y}\right\rangle \pm \sigma$ & $\begin{array}{c}a, \mathrm{~mm} \\
(\text { at } 250 \mathrm{GeV})\end{array}$ & $w$ \\
\hline 0.01 & -0.0151 & $1.000 \pm 0.0000$ & 0.008 & 0.135 \\
0.02 & -0.0150 & $1.000 \pm 0.0001$ & 0.016 & 0.133 \\
0.03 & -0.0149 & $0.999 \pm 0.0003$ & 0.024 & 0.130 \\
0.04 & -0.0146 & $0.998 \pm 0.0011$ & 0.032 & 0.125 \\
0.05 & -0.0144 & $0.999 \pm 0.0007$ & 0.040 & 0.120 \\
0.06 & -0.0141 & $0.994 \pm 0.0029$ & 0.048 & 0.113 \\
0.08 & -0.0134 & $0.923 \pm 0.0525$ & 0.063 & 0.098 \\
0.10 & -0.0124 & $0.994 \pm 0.0042$ & 0.079 & 0.082 \\
0.15 & -0.0100 & $0.987 \pm 0.0089$ & 0.119 & 0.044 \\
0.20 & -0.0078 & $0.978 \pm 0.0157$ & 0.159 & 0.018 \\
0.30 & -0.0046 & $0.951 \pm 0.0351$ & 0.238 & 0.001 \\
0.40 & -0.0029 & $-0.082 \pm 0.1742$ & 0.317 & 0.000 \\
\hline
\end{tabular}

Table 1: SPINK tracking results. $a_{0}$ is the initial transverse amplitude at $157 \mathrm{GeV}$, $\delta \nu_{y}$ is the vertical beam-beam tune shift, $S_{y}$ is the vertical spin component at the interaction point, $a$ is the particle transverse amplitude at storage energy, and $w$ is the weighting used in computing the distribution average and rms spin. 


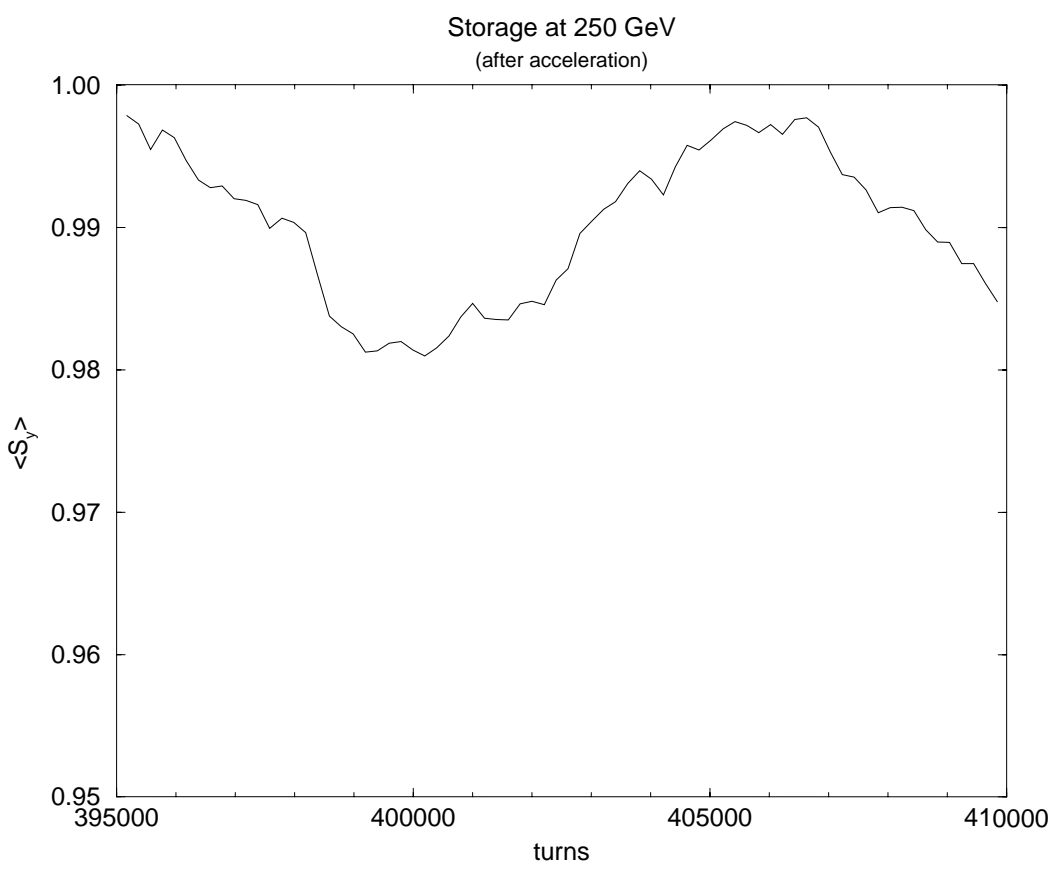

Figure 6: Average vertical spin component versus turn number for storage at $250 \mathrm{GeV}$.

using thin quadrupole lenses associated with each quadrupole of the storage ring. Since the beam-beam interaction is also shifting the betatron tune, it may enhance the depolarization of some fraction of the beam whose tune is shifted toward $1 / 6$ or $5 / 6$.

To explore this effect, a small number of particles with various initial transverse amplitudes were tracked for 5,000 turns, with Snakes on, and at fixed energy corresponding to $G \gamma=261$ close to the strong intrinsic spin resonance at $G \gamma=261.18=$ $232+\nu_{y}$. Results are shown in Fig.7. The curves on the right show the depolarization in the bare machine (beam-beam off) as a function of tune variation (the quantity $\delta q$ shown is a detune parameter used in $S P I N K$ ). The curves on the left represent the same particles with beam-beam on, labeled according to their initial displacement.

The beam-beam-off curves show an enhanced depolarization with a fine structure near $\Delta \nu=1 / 6=0.1666$, where $\Delta \nu$ represents the fractional part of the vertical tune. The base line is the depolarization occurring at that energy and the nominal tune. The beam-beam-on curves show a more enhanced depolarization occurring at higher values of $\Delta \nu$, since the beam-beam interaction effectively increases the tune shift by a different amount for each individual particle. The $\Delta \nu$ values on the horizontal axis are the bare machine values. Note that the peaks on the left cluster around $\delta q=0$, where the corresponding nominal bare tune fractional part is exactly 0.18 . 


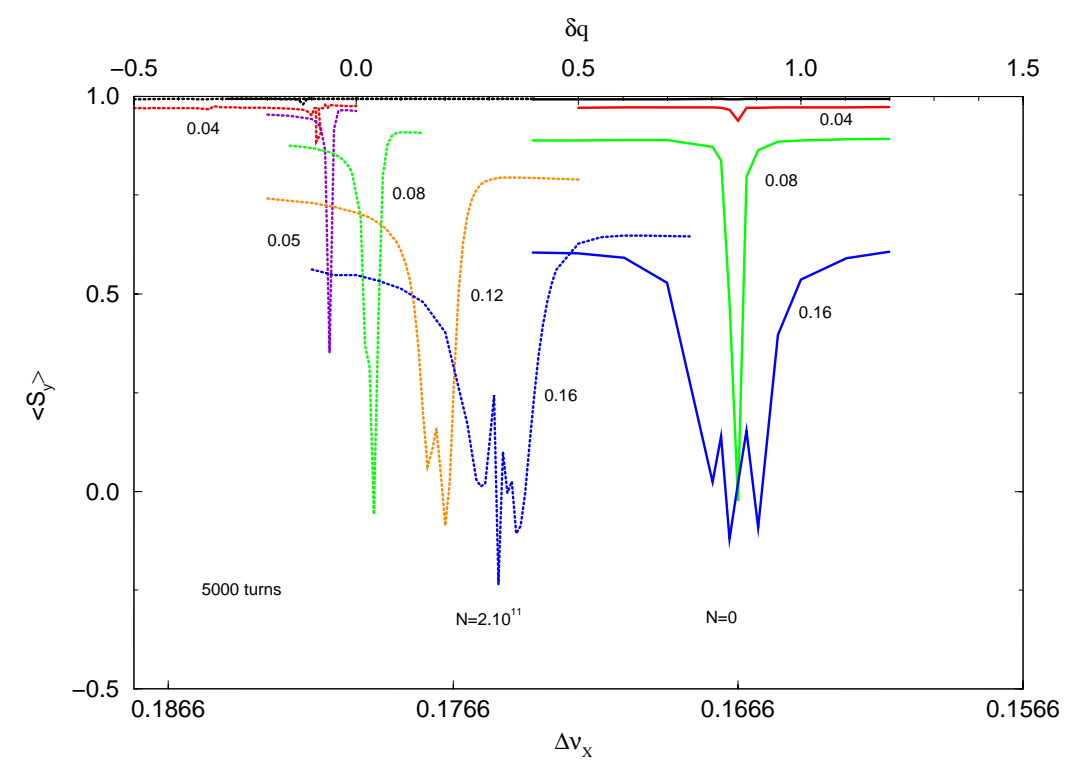

Figure 7: Spin versus tune for particles of various amplitudes. Curves on right are without beam-beam, curves on left are with beam-beam.

\section{Concluding Remarks}

As expected, particle tracking using SPINK shows that the beam-beam effect for reasonable RHIC beam parameters does not enhance the resonances in RHIC enough to generate depolarization during storage at $250 \mathrm{GeV}$. This is in qualitative agreement with the results found by Batygin and Katayama[4] using a simple model for RHIC. For other possible RHIC collision energies, especially near $200 \mathrm{GeV}$, the situation will need to be studied in further detail. It also will be important to include other sources of tune spread and tune modulation into SPINK as well.

\section{References}

[1] A. Luccio, "Spin Tracking Code in RHIC (SPINK)," Proc. Adriatico Conf., Trieste, Italy, Dec. 1995, World Scientific.

[2] A. Luccio, "Angles from Spin Matrices," BNL report AGS/RHIC/SN-039, October 1996.

[3] L.H. Thomas, Phil. Mag. 3, 1 (1927); V. Bargmann, L. Michel, V.L. Telegdi, Phys. Rev. Lett. 2, 435 (1959).

[4] Y. Batygin and T. Katayama, "Numerical Study of Spin Depolarization in RHIC due to Beam-beam Collision," BNL report AGS/RHIC/SN-053, April 25, 1997. 